\title{
LIGHTS OUT! ON GRAPH PRODUCTS OVER THE RING OF INTEGERS MODULO $k^{*}$
}

\author{
RYAN MUNTER ${ }^{\dagger}$ AND TRAVIS PETERS ${ }^{\dagger}$
}

\begin{abstract}
LIGHTS OUT! is a game played on a finite, simple graph. The vertices of the graph are the lights, which may be on or off, and the edges of the graph determine how neighboring vertices turn on or off when a vertex is pressed. Given an initial configuration of vertices that are on, the object of the game is to turn all the lights out. The traditional game is played over $\mathbb{Z}_{2}$, where the vertices are either lit or unlit, but the game can be generalized to $\mathbb{Z}_{k}$, where the lights have different colors. Previously, the game was investigated on Cartesian product graphs over $\mathbb{Z}_{2}$. We extend this work to $\mathbb{Z}_{k}$ and investigate two other fundamental graph products, the direct (or tensor) product and the strong product. We provide conditions for which the direct product graph and the strong product graph are solvable based on the factor graphs, and we do so using both open and closed neighborhood switching over $\mathbb{Z}_{k}$.
\end{abstract}

Key words. Matrix, Determinant, Graph, Graph product, LIGHTS OUT.

AMS subject classifications. 05C50, 15A15, 15B33, $05 \mathrm{C} 76$.

1. Introduction. The game LIGHTS OUT! is played on a finite, simple graph $G$. The vertices of $G$ are labeled using elements of $\mathbb{Z}_{2}=\{0,1\}$, where 1 denotes 'on' and 0 denotes 'off.' Each vertex can be thought of as a button with a light. Pressing a vertex toggles that vertex and all adjacent vertices between on and off. Given an initial configuration of buttons that are on, the object of the game is to turn all the lights out. The graph $G$ is said to be universally solvable if every initial configuration of lights is solvable (can be transformed to the all off state by a sequence of presses). Giffen and Parker [4] generalized the game to $\mathbb{Z}_{k}, k \geq 2$. Here, the vertices of $G$ are labeled using elements of $\mathbb{Z}_{k}=\{0,1,2, \ldots, k-1\}$, and we can think of these elements as representing different colors of lights. Pressing a vertex increases the label of that vertex and the vertices adjacent to it by 1 modulo $k$. The game is won when every vertex has label 0 .

Let $G=(V, E)$ be a simple graph of order $n$. For each $v \in V$, the open neighborhood $N(v)$ of $v$ is the set of vertices adjacent to $v, N(v)=\{u \in V:(u, v) \in E\}$. The closed neighborhood $N[v]$ of $v$ is the open neighborhood along with $v$ itself, $N[v]=N(v) \cup\{v\}$. In the traditional game, pressing a vertex $v$ toggles $v$ as well as the vertices adjacent to $v$. This is called closed neighborhood switching. In a variation of the game, pressing a vertex $v$ toggles the vertices adjacent to $v$ but not $v$ itself. This is called open neighborhood switching.

The graph $G$ is closed universally solvable if every initial configuration is solvable using closed neighborhood switching. The graph $G$ is open universally solvable if every initial configuration is solvable using open neighborhood switching. Throughout the paper, we denote by $A(G)$ the adjacency matrix of $G$. Then, $A(G)$ is the open neighborhood matrix of $G$, and $A(G)+I_{n}$, where $I_{n}$ is the $n \times n$ identity matrix, is the closed neighborhood matrix of $G$.

TheOREM 1.1 ([8]). A graph $G$ is open (closed) universally solvable over $\mathbb{Z}_{k}(k \geq 2)$ if and only if $A(G)$ $\left(A(G)+I_{n}\right)$ is invertible over $\mathbb{Z}_{k}$

${ }^{*}$ Received by the editors on August 17, 2020. Accepted for publication on May 1, 2021. Handling Editor: Bryan Shader. Corresponding Author: Travis Peters.

${ }^{\dagger}$ Department of Mathematics, College of Saint Benedict \& Saint John's University, Saint Joseph, Minnesota 56374, USA (rmunter001@csbsju.edu, tpeters001@csbsju.edu). 
Electronic Journal of Linear Algebra, ISSN 1081-3810

A publication of the International Linear Algebra Society

Volume 37, pp. 416-424, May 2021.

We approach the problem of determining whether or not a graph $G$ is open (closed) universally solvable over $\mathbb{Z}_{k}$ by computing the determinant of the adjacency matrix $A(G)$ (the closed neighborhood matrix $\left.A(G)+I_{n}\right)$. If $\mathbb{Z}_{k}$ is a field, then a matrix $A \in M_{n}\left(\mathbb{Z}_{k}\right)$ is invertible if and only if $\operatorname{det}(A) \neq 0$. However, this result does not hold in arbitrary commutative rings. An element $r$ of a commutative ring $R$ is a unit if it has a multiplicative inverse, i.e. there exists $s \in R$ such that $r s=1_{R}$. The set of units of $\mathbb{Z}_{k}$ consists of the nonzero elements of $\mathbb{Z}_{k}$ relatively prime to $k$. The set of nonzero nonunits (zero divisors) of $\mathbb{Z}_{k}$ consists of the nonzero elements of $\mathbb{Z}_{k}$ not relatively prime to $k$, so that every nonzero element of $\mathbb{Z}_{k}$ is a zero divisor or a unit (but not both). For an arbitrary ring of integers modulo $k$, we need the following result.

Theorem 1.2 ([2], Corollary 2.21). Let $A \in M_{n}(R)$, where $R$ is a commutative ring. Then, $A$ is invertible if and only if $\operatorname{det}(A)$ is a unit of $R$.

Goldwasser et al. [5, 6] investigated the game applied to rectangular grid graphs. They used Fibonacci polynomials to determine the pairs $(m, n)$ for which the grid graph $G_{m, n}$ is open and closed universally solvable over $\mathbb{Z}_{2}$. The grid graph $G_{m, n}$ is simply the Cartesian product graph $P_{m} \square P_{n}$ of the path graphs $P_{m}$ and $P_{n}$. In [9], Peters et al. generalized the game to the Cartesian product graph $G \square H$ of arbitrary finite, simple graphs $G$ and $H$, and they provided conditions for universal solvability over $\mathbb{Z}_{2}$ using both open and closed neighborhood switching. In this paper, we investigate the game applied to two other fundamental graph products, the direct (or tensor) product and the strong product. In particular, we provide conditions for universal solvability over $\mathbb{Z}_{k}$ of the direct product graph and the strong product graph in terms of the factor graphs using both open and closed neighborhood switching (see Sections 3 and 4). As such, it is useful to know the solvability of common graph families since these well-known graphs are often the factors of the product (see [4] for a discussion of the solvability of paths, cycles, and complete bipartite graphs over $\mathbb{Z}_{k}$ ). Finally, in Section 5 , we extend previous results on the Cartesian product graph over $\mathbb{Z}_{2}$ to $\mathbb{Z}_{k}$.

2. Preliminaries. The three fundamental graph products (Cartesian, direct, and strong) have the same vertex set but different edge sets. If $G$ and $H$ are the factors of the product, then the vertex set of the product graph is the Cartesian product $V(G) \times V(H)=\{(g, h): g \in V(G)$ and $h \in V(H)\}$. The edge set of the Cartesian product $G \square H$ is $E(G \square H)=\left\{(g, h)\left(g^{\prime}, h^{\prime}\right): g=g^{\prime}, h h^{\prime} \in E(H)\right.$ or $\left.h=h^{\prime}, g g^{\prime} \in E(G)\right\}$. The edge set of the direct product $G \times H$ is $E(G \times H)=\left\{(g, h)\left(g^{\prime}, h^{\prime}\right): g g^{\prime} \in E(G)\right.$ and $\left.h h^{\prime} \in E(H)\right\}$. Finally, the edge set of the strong product $G \otimes H$ is $E(G \otimes H)=E(G \square H) \cup E(G \times H)$. Figure 1 displays the Cartesian product $P_{4} \square P_{3}$, direct product $P_{4} \times P_{3}$, and strong product $P_{4} \otimes P_{3}$. For a thorough discussion of graph products, see [7].
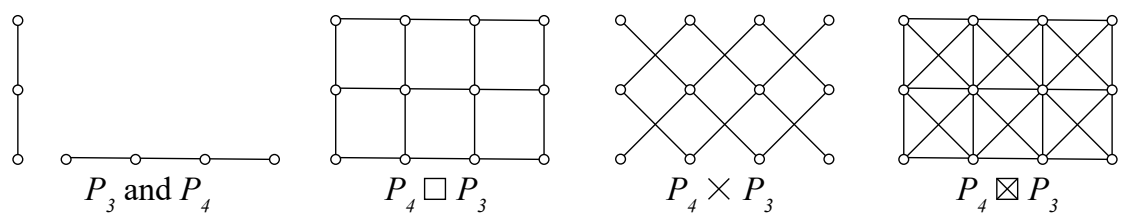

Figure 1. The factor graphs $P_{3}$ and $P_{4}$ along with the Cartesian product $P_{4} \square P_{3}$, direct product $P_{4} \times P_{3}$, and strong product $P_{4} \otimes P_{3}$ graphs. 
Electronic Journal of Linear Algebra, ISSN 1081-3810

The adjacency matrix of $G \square H$ is given by

$$
\begin{aligned}
A(G \square H) & =A(G) \oplus A(H) \\
& =A(G) \otimes I_{|H|}+I_{|G|} \otimes A(H),
\end{aligned}
$$

where $\oplus$ denotes a Kronecker sum of matrices and $\otimes$ denotes a Kronecker product of matrices (see [10]). For example, consider the graphs $G=P_{4}$ and $H=P_{3}$. Then

$$
A\left(P_{4}\right)=\left[\begin{array}{llll}
0 & 1 & 0 & 0 \\
1 & 0 & 1 & 0 \\
0 & 1 & 0 & 1 \\
0 & 0 & 1 & 0
\end{array}\right],
$$

and

$$
A\left(P_{3}\right)=\left[\begin{array}{lll}
0 & 1 & 0 \\
1 & 0 & 1 \\
0 & 1 & 0
\end{array}\right] .
$$

Moreover,

$$
\begin{aligned}
A\left(P_{4} \square P_{3}\right) & =A\left(P_{4}\right) \oplus A\left(P_{3}\right) \\
& =A\left(P_{4}\right) \otimes I_{3}+I_{4} \otimes A\left(P_{3}\right) \\
& =\left[\begin{array}{cccc}
A\left(P_{3}\right) & I_{3} & O_{3} & O_{3} \\
I_{3} & A\left(P_{3}\right) & I_{3} & O_{3} \\
O_{3} & I_{3} & A\left(P_{3}\right) & I_{3} \\
O_{3} & O_{3} & I_{3} & A\left(P_{3}\right)
\end{array}\right],
\end{aligned}
$$

where $I_{3}$ denotes the $3 \times 3$ identity matrix and $O_{3}$ denotes the $3 \times 3$ matrix of zeros. The adjacency matrix of $G \times H$ is given by

$$
A(G \times H)=A(G) \otimes A(H) .
$$

For $G=P_{4}$ and $H=P_{3}$, we have

$$
\begin{aligned}
A\left(P_{4} \times P_{3}\right) & =A\left(P_{4}\right) \otimes A\left(P_{3}\right) \\
& =\left[\begin{array}{cccc}
O_{3} & A\left(P_{3}\right) & O_{3} & O_{3} \\
A\left(P_{3}\right) & O_{3} & A\left(P_{3}\right) & O_{3} \\
O_{3} & A\left(P_{3}\right) & O_{3} & A\left(P_{3}\right) \\
O_{3} & O_{3} & A\left(P_{3}\right) & O_{3}
\end{array}\right] .
\end{aligned}
$$

Finally, the adjacency matrix of $G \otimes H$ is given by

$$
\begin{aligned}
A(G \otimes H) & =A(G) \oplus A(H)+A(G) \otimes A(H) \\
& =\left(A(G)+I_{|G|}\right) \otimes\left(A(H)+I_{|H|}\right)-I_{|G|} \otimes I_{|H|} .
\end{aligned}
$$


For $G=P_{4}$ and $H=P_{3}$, we have

$$
\begin{aligned}
A\left(P_{4} \otimes P_{3}\right)= & A\left(P_{4}\right) \oplus A\left(P_{3}\right)+A\left(P_{4}\right) \otimes A\left(P_{3}\right) \\
= & \left(A\left(P_{4}\right)+I_{4}\right) \otimes\left(A\left(P_{3}\right)+I_{3}\right)-I_{4} \otimes I_{3} \\
= & {\left[\begin{array}{cccc}
A\left(P_{3}\right) & A\left(P_{3}\right)+I_{3} & O_{3} & O_{3} \\
A\left(P_{3}\right)+I_{3} & A\left(P_{3}\right) & A\left(P_{3}\right)+I_{3} & O_{3} \\
O_{3} & A\left(P_{3}\right)+I_{3} & A\left(P_{3}\right) & A\left(P_{3}\right)+I_{3} \\
O_{3} & O_{3} & A\left(P_{3}\right)+I_{3} & A\left(P_{3}\right)
\end{array}\right] . }
\end{aligned}
$$

Throughout the paper, we will use the following result concerning the determinant of a block matrix with blocks that mutually commute.

TheOREM 2.1 ([11]). Let $R$ be a commutative subring of $M_{n}(F)$, where $F$ is a field (or a commutative ring), and let $\mathbf{M} \in M_{m}(R)$. Then

$$
\operatorname{det}_{F} \mathbf{M}=\operatorname{det}_{F}\left(\operatorname{det}_{R} \mathbf{M}\right)
$$

For example, if $\mathbf{M}=\left[\begin{array}{ll}\mathbf{A} & \mathbf{B} \\ \mathbf{C} & \mathbf{D}\end{array}\right]$ where $\mathbf{A}, \mathbf{B}, \mathbf{C}$, and $\mathbf{D}$ are $n \times n$ matrices over $F$ which mutually commute, then Theorem 2.1 says

$$
\operatorname{det}_{F} \mathbf{M}=\operatorname{det}_{F}(\mathbf{A D}-\mathbf{B C}) .
$$

The notation $\operatorname{det}_{F} \mathbf{M}$ indicates that the determinant of $\mathbf{M}$ is an element of $F$.

3. Direct (tensor) products. Let $G$ and $H$ be finite, simple graphs, with $|G|=m,|H|=p$, and let $n=m p$. Let $B=\left[b_{i j}\right]=A(G)$, the adjacency matrix of $G$, and let $C=A(H)$, the adjacency matrix of $H$. We make the following observation.

ObSERvation 3.1. The adjacency matrix of $G \times H$ is

$$
A(G \times H)=\left[\begin{array}{cccc}
O_{p} & b_{12} C & \ldots & b_{1 m} C \\
b_{21} C & O_{p} & & \vdots \\
\vdots & & \ddots & b_{(m-1) m} C \\
b_{m 1} C & \ldots & b_{m(m-1)} C & O_{p}
\end{array}\right],
$$

where $b_{i j} C$ is either the $p \times p$ matrix $C$ or the $p \times p$ matrix of zeros $O_{p}$ as $b_{i j} \in \mathbb{Z}_{2}$ for all $1 \leq i, j \leq m$. Note that $b_{i i}=0$ for all $1 \leq i \leq m$ as $B$ is the adjacency matrix of $G$. The rows and columns could be permuted to get an adjacency matrix with $p m \times m$ blocks consisting of $B$ and $O_{m}$.

By Observation 3.1, $A(G \times H)$ is a block matrix consisting of the blocks $C=A(H)$ and the $p \times p$ matrix of zeros $O_{p}$. These two blocks along with the identity matrix $I_{p}$ mutually commute, so the next result follows from Theorem 2.1. We let $p_{A}(x)$ denote the characteristic polynomial of the matrix $A \in M_{n}(F)$.

LEMmA 3.2. Let $G$ and $H$ be finite, simple graphs, with $|G|=m,|H|=p$, and let $n=m p$. Let $B=A(G)$ and $C=A(H)$. Then

$$
\operatorname{det}[A(G \times H)]=\operatorname{det}\left[p_{\left(-B x+I_{m} x\right)}(C)\right],
$$


and

$$
\operatorname{det}\left[A(G \times H)+I_{n}\right]=\operatorname{det}\left[p_{\left(-B x+I_{m}(x-1)\right)}(C)\right]
$$

If both $G$ and $H$ are closed universally solvable, $G \times H$ may or may not be closed universally solvable. For example, $P_{3}, P_{4}$, and $C_{5}$ are all closed universally solvable over $\mathbb{Z}_{2}$. However, $P_{3} \times C_{5}$ is closed universally solvable over $\mathbb{Z}_{2}$, while $P_{4} \times C_{5}$ is not closed universally solvable over $\mathbb{Z}_{2}$. If just $G$ or just $H$ is closed universally solvable, $G \times H$ may or may not be closed universally solvable. The graphs $P_{3}$ and $P_{6}$ are closed universally solvable over $\mathbb{Z}_{2}$, and the graph $P_{8}$ is not closed universally solvable over $\mathbb{Z}_{2}$. While $P_{3} \times P_{8}$ is closed universally solvable over $\mathbb{Z}_{2}, P_{6} \times P_{8}$ is not closed universally solvable over $\mathbb{Z}_{2}$. The following lemma will be used to establish the result that if $G$ and $H$ are not closed universally solvable over $\mathbb{Z}_{2}$, then $G \times H$ is not closed universally solvable over $\mathbb{Z}_{2}$. It turns out that we cannot extend this result to $\mathbb{Z}_{k}$, for $k \geq 3$. For example, $C_{3}$ and $W_{4}$, the wheel graph of order 4 , are not closed universally solvable over $\mathbb{Z}_{3}$. However, $C_{3} \times W_{4}$ is closed universally solvable over $\mathbb{Z}_{3}$.

LEMMA 3.3 ([9]). Let $G$ and $H$ be finite, simple graphs, with $|G|=m,|H|=p$, and let $n=m p$. Let $B=$ $A(G)$ and $C=A(H)$. Then, $p_{\left(-B x+I_{m}(x-1)\right)}(C)$ is singular if and only if $\operatorname{gcd}\left(p_{\left(-B x+I_{m}(x-1)\right)}(x), p_{C}(x)\right) \neq 1$.

TheOrem 3.4. Let $G$ and $H$ be finite, simple graphs, with $|G|=m,|H|=p$, and let $n=m p$. Let $B=A(G)$ and $C=A(H)$. If $G$ and $H$ are not closed universally solvable over $\mathbb{Z}_{2}$, then $G \times H$ is not closed universally solvable over $\mathbb{Z}_{2}$.

Proof. Suppose $G$ and $H$ are not closed universally solvable over $\mathbb{Z}_{2}$. Then, $\operatorname{det}\left(B+I_{m}\right)=\operatorname{det}\left(C+I_{p}\right)=$ 0 . Moreover, $p_{\left(-B x+I_{m}(x-1)\right)}(1)=\operatorname{det}\left[1 \cdot I_{m}-\left(-B \cdot 1+I_{m}(1-1)\right)\right]=\operatorname{det}\left[B+I_{m}\right]=0$. In other words, 1 is a root of the characteristic polynomial of $-B x+I_{m}(x-1)$ and $x+1$ is a factor of $p_{\left(-B x+I_{m}(x-1)\right)}(x)$. In addition, $p_{C}(1)=\operatorname{det}\left[1 \cdot I_{p}-C\right]=\operatorname{det}\left[C+I_{p}\right]=0$. Therefore, 1 is a root of the characteristic polynomial of $C$, and $x+1$ is a factor of $p_{C}(x)$. Hence, $x+1$ is a common divisor of $p_{C}(x)$ and $p_{\left(-B x+I_{m}(x-1)\right)}(x)$. By Lemma 3.3, $p_{\left(-B x+I_{m}(x-1)\right)}(C)$ is singular, meaning that $G \times H$ is not closed universally solvable over $\mathbb{Z}_{2}$.

Next, we turn to open neighborhood switching for $G \times H$. We will need the following important result concerning the determinant of a tensor product of matrices.

Theorem 3.5 ([11]). Let $F$ be a field (or a commutative ring), $\mathbf{P} \in M_{m}(F)$, and $\mathbf{Q} \in M_{n}(F)$. Then

$$
\operatorname{det}_{F}(\mathbf{P} \otimes \mathbf{Q})=\left(\operatorname{det}_{F} \mathbf{P}\right)^{n}\left(\operatorname{det}_{F} \mathbf{Q}\right)^{m}
$$

For commutative rings, we mention the following known result about units.

REMARK 3.6. Let $R$ be a commutative ring. Then, $a, b \in R$ are units in $R$ if and only if $a b$ is a unit in $R$.

TheOrem 3.7. Let $G$ and $H$ be finite, simple graphs, with $|G|=m,|H|=p$, and let $n=m p$. Let $B=A(G)$ and $C=A(H)$. Then, $G \times H$ is open universally solvable over $\mathbb{Z}_{k}(k \geq 2)$ if and only if $G$ and $H$ are open universally solvable over $\mathbb{Z}_{k}$.

Proof. Suppose $G \times H$ is open universally solvable over $\mathbb{Z}_{k}, k \geq 2$. By Theorem $3.5, \operatorname{det}[A(G \times H)]=$ $\operatorname{det}[B \otimes C]=(\operatorname{det} B)^{p}(\operatorname{det} C)^{m}=r$ is a unit in $\mathbb{Z}_{k}$. Hence, $\operatorname{det}(B)$ and $\operatorname{det}(C)$ are units in $\mathbb{Z}_{k}$ by Remark 3.6. Therefore, $G$ and $H$ are open universally solvable over $\mathbb{Z}_{k}$. 
Electronic Journal of Linear Algebra, ISSN 1081-3810

A publication of the International Linear Algebra Society

Volume 37, pp. 416-424, May 2021

Conversely, suppose $G$ and $H$ are open universally solvable over $\mathbb{Z}_{k}$. Then, $\operatorname{det}(B)$ and $\operatorname{det}(C)$ are units in $\mathbb{Z}_{k}$. By Theorem 3.5, $\operatorname{det}[A(G \times H)]=\operatorname{det}[B \otimes C]=(\operatorname{det} B)^{p}(\operatorname{det} C)^{m}$, and this must be a unit in $\mathbb{Z}_{k}$ by Remark 3.6. Thus, $G \times H$ is open universally solvable over $\mathbb{Z}_{k}$.

4. Strong products. Let $G$ and $H$ be finite, simple graphs, with $|G|=m,|H|=p$, and let $n=m p$. Let $B=\left[b_{i j}\right]=A(G)$, the adjacency matrix of $G$, and let $C=A(H)$, the adjacency matrix of $H$. We make the following observation.

OBSERVATION 4.1. The adjacency matrix of $G \otimes H$ is

$$
A(G \otimes H)=\left[\begin{array}{cccc}
C & b_{12}\left(C+I_{p}\right) & \cdots & b_{1 m}\left(C+I_{p}\right) \\
b_{21}\left(C+I_{p}\right) & C & \ddots & b_{(m-1) m}\left(C+I_{p}\right) \\
\vdots & & b_{m(m-1)}\left(C+I_{p}\right) & C \\
b_{m 1}\left(C+I_{p}\right) & \ldots &
\end{array}\right],
$$

where $b_{i j}\left(C+I_{p}\right)$ is either the $p \times p$ matrix $C+I_{p}$ or the $p \times p$ matrix of zeros as $b_{i j} \in \mathbb{Z}_{2}$ for all $1 \leq i, j \leq m$. Note that $b_{i i}=0$ for all $1 \leq i \leq m$ as $B$ is the adjacency matrix of $G$. The rows and columns could be permuted to get an adjacency matrix with $p=m$ blocks with $B$ on the main diagonal.

By Observation 4.1, $A(G \otimes H)$ is a block matrix consisting of the blocks $C=A(H)$, the $p \times p$ matrix $\left(C+I_{p}\right)$, and the $p \times p$ matrix of zeros $O_{p}$. These three blocks mutually commute, so the next result follows from Theorem 2.1. We let $p_{A}(x)$ denote the characteristic polynomial of the matrix $A \in M_{n}(F)$.

LEMmA 4.2. Let $G$ and $H$ be finite, simple graphs, with $|G|=m,|H|=p$, and let $n=m p$. Let $B=A(G)$ and $C=A(H)$. Then

$$
\operatorname{det}[A(G \otimes H)]=\operatorname{det}\left[p_{(-B(x+1))}(C)\right]
$$

and

$$
\operatorname{det}\left[A(G \otimes H)+I_{n}\right]=\operatorname{det}\left[p_{(-B x)}\left(C+I_{p}\right)\right]
$$

We will need the following lemma to establish conditions for universal solvability for closed neighborhood switching on the strong product graph.

LemmA 4.3. Let $A \in M_{n}\left(\mathbb{Z}_{k}\right)$ and let $x \in \mathbb{Z}_{k}, k \geq 2$. Then $p_{(-A x)}(x)=\operatorname{det}\left(A+I_{n}\right) \cdot x^{n}$.

Proof. Observe that

$$
\begin{aligned}
p_{(-A x)}(x) & =\operatorname{det}\left[x I_{n}-(-A x)\right] \\
& =\operatorname{det}\left[x\left(A+I_{n}\right)\right] \\
& =x^{n} \cdot \operatorname{det}\left[A+I_{n}\right] .
\end{aligned}
$$

We now provide conditions for which $G \otimes H$ is closed universally solvable over $\mathbb{Z}_{k}, k \geq 2$.

Theorem 4.4. Let $G$ and $H$ be finite, simple graphs, with $|G|=m,|H|=p$, and let $n=m p$. Let $B=A(G)$ and $C=A(H)$. Then, $G \otimes H$ is closed universally solvable over $\mathbb{Z}_{k}(k \geq 2)$ if and only if $G$ and $H$ are closed universally solvable over $\mathbb{Z}_{k}$. 
Proof. Suppose $G \otimes H$ is closed universally solvable over $\mathbb{Z}_{k}, k \geq 2$. Then, $\operatorname{det}\left[A(G \otimes H)+I_{n}\right]=r$ is a unit in $\mathbb{Z}_{k}$. By Lemmas 4.2 and 4.3 ,

$$
\begin{aligned}
r & =\operatorname{det}\left[A(G \otimes H)+I_{n}\right] \\
& =\operatorname{det}\left[p_{(-B x)}\left(C+I_{p}\right)\right] \\
& =\operatorname{det}\left[\operatorname{det}\left(B+I_{m}\right) \cdot\left(C+I_{p}\right)^{m}\right] \\
& =\left[\operatorname{det}\left(B+I_{m}\right)\right]^{p} \cdot\left[\operatorname{det}\left(C+I_{p}\right)\right]^{m} .
\end{aligned}
$$

Therefore, $\left[\operatorname{det}\left(B+I_{m}\right)\right]^{p}$ and $\left[\operatorname{det}\left(C+I_{p}\right)\right]^{m}$ are units in $\mathbb{Z}_{k}$ by Remark 3.6, and therefore $\operatorname{det}\left(B+I_{m}\right)$ and $\operatorname{det}\left(C+I_{p}\right)$ are units in $\mathbb{Z}_{k}$. It follows that $G$ and $H$ are closed universally solvable over $\mathbb{Z}_{k}$.

Conversely, suppose $G$ and $H$ are closed universally solvable over $\mathbb{Z}_{k}$. Then, $\operatorname{det}\left(B+I_{m}\right)$ and $\operatorname{det}\left(C+I_{p}\right)$ are units in $\mathbb{Z}_{k}$. By Remark 3.6, $\left[\operatorname{det}\left(B+I_{m}\right)\right]^{p}$ and $\left[\operatorname{det}\left(C+I_{p}\right)\right]^{m}$ are also units in $\mathbb{Z}_{k}$. By Lemmas 4.2 and 4.3 and Remark 3.6, $\operatorname{det}\left[A(G \otimes H)+I_{n}\right]=\operatorname{det}\left[p_{(-B x)}\left(C+I_{p}\right)\right]=\left[\operatorname{det}\left(B+I_{m}\right)\right]^{p} \cdot\left[\operatorname{det}\left(C+I_{p}\right)\right]^{m}$ is a unit in $\mathbb{Z}_{k}$. Hence, $G \otimes H$ is closed universally solvable over $\mathbb{Z}_{k}$.

This elegant result is quite surprising given that $G \square H$ and $G \times H$ need not be closed universally solvable when $G$ and $H$ are both closed universally solvable.

Next, we turn to open neighborhood switching for $G \otimes H$. If $G$ and $H$ are both open universally solvable, $G \otimes H$ may or may not be open universally solvable. For example, $P_{4}, K_{4}$, and $W_{6}$, the wheel graph of order 6 , are all open universally solvable over $\mathbb{Z}_{2}$. However, $P_{4} \otimes K_{4}$ is open universally solvable over $\mathbb{Z}_{2}$, while $W_{6} \otimes P_{4}$ is not open universally solvable over $\mathbb{Z}_{2}$. If just $G$ or just $H$ is open universally solvable, $G \otimes H$ may or may not be open universally solvable. For example, $P_{3}$ and $P_{9}$ are not open universally solvable over $\mathbb{Z}_{2}$. However, $P_{4} \otimes P_{3}$ is open universally solvable over $\mathbb{Z}_{2}$, but $P_{4} \otimes P_{9}$ is not open universally solvable over $\mathbb{Z}_{2}$. We will show that if $G$ and $H$ are not open universally solvable over $\mathbb{Z}_{m}$, where $m$ is a power of a prime, then $G \otimes H$ is not open universally solvable over $\mathbb{Z}_{m}$.

Galovich [3] investigated unique factorization rings which are not integral domains, i.e., which contain zero divisors. In a unique factorization ring, every nonzero nonunit has a unique factorization into irreducibles (see [3]). Galovich showed that $\mathbb{Z}_{m}$ is a unique factorization ring if and only if $m$ is a power of a prime, a result that was originally proven by Billis [1]. Recall that an element $r$ of an arbitrary ring is nilpotent if there exists some positive integer $n$ such that $r^{n}=0$.

We make the following observation.

OBSERVATION 4.5. If an element of a commutative ring $R$ is nilpotent, then the element is not a unit of $R$.

TheOREM 4.6. Let $G$ and $H$ be finite, simple graphs, with $|G|=m,|H|=p$, and let $n=m p$. Let $B=A(G)$ and $C=A(H)$. If $G$ and $H$ are not open universally solvable over $\mathbb{Z}_{m}$, where $m$ is a power of a prime, then $G \otimes H$ is not open universally solvable over $\mathbb{Z}_{m}$.

Proof. Suppose $G$ and $H$ are not open universally solvable over $\mathbb{Z}_{m}$, where $m$ is a power of a prime. Then, $\operatorname{det}(B)=d_{1}$ and $\operatorname{det}(C)=d_{2}$, where $d_{1}$ and $d_{2}$ are nonunits of $\mathbb{Z}_{m}$. Moreover, $p_{-(B(x+1))}(0)=$ $\operatorname{det}\left(0 \cdot I_{m}+B(0+1)\right)=\operatorname{det}(B)=d_{1}$. Therefore, $p_{-(B(x+1))}(x)=q(x)+d_{1}$ for some $q(x) \in \mathbb{Z}_{m}[x]$ which has a factor of $x$. As every nonunit in a unique factorization ring is nilpotent [3], $d_{1}$ is nilpotent. Hence, there exists a positive integer $j$ such that $d_{1}^{j}=0$. Then, $\left(p_{-(B(x+1))}(x)\right)^{j}=\left(q(x)+d_{1}\right)^{j}=s(x)$ for some $s(x) \in \mathbb{Z}_{m}[x]$ which has a factor of $x$. Then, $s(x)=x \cdot t(x)$ for some $t(x) \in \mathbb{Z}_{m}[x]$. Hence, 
$\operatorname{det}\left[\left(p_{-(B(x+1))}(C)\right)^{j}\right]=\operatorname{det}[C \cdot t(C)]$. Therefore, $\left[\operatorname{det}\left(p_{-(B(x+1))}(C)\right)\right]^{j}=\operatorname{det}(C) \cdot \operatorname{det}(t(C))=d_{2} \cdot \operatorname{det}(t(C))$. Since $d_{2}$ is a nonunit, and therefore nilpotent, there exists a positive integer $k$ such that $d_{2}^{k}=0$. So, $\left[\operatorname{det}\left(p_{-(B(x+1))}(C)\right)\right]^{j k}=d_{2}^{k} \cdot \operatorname{det}(t(C))^{k}=0$, meaning that $\operatorname{det}\left[\left(p_{-(B(x+1))}(C)\right)\right]$ is nilpotent. By Observation $4.5, \operatorname{det}\left[\left(p_{-(B(x+1))}(C)\right)\right]$ is a nonunit of $\mathbb{Z}_{m}$. Thus, $G \otimes H$ is not open universally solvable over $\mathbb{Z}_{m}$.

Theorem 4.6 does not hold for rings of integers modulo $k$ which are not unique factorization rings, i.e. when $k$ is not a power of a prime. For example, $W_{3}$, the wheel graph of order 3 , and $K_{4}$ are not open universally solvable over $\mathbb{Z}_{6}$, but $W_{3} \otimes K_{4}$ is open universally solvable over $\mathbb{Z}_{6}$.

5. Cartesian products revisited. In characterizing the direct product and the strong product, we were able to provide conditions for universal solvability in terms of the factor graphs. The same can be done for open neighborhood switching for the Cartesian product.

Unfortunately, for closed neighborhood switching, we cannot say anything about the universal solvability of $G \square H$ in terms of the factor graphs. If $G$ and $H$ are both closed universally solvable, $G \square H$ may or may not be closed universally solvable. For example, the graphs $P_{4}, P_{6}$, and $C_{5}$ are closed universally solvable over $\mathbb{Z}_{2}$. While $P_{4} \square P_{6}$ is closed universally solvable over $\mathbb{Z}_{2}, P_{4} \square C_{5}$ is not closed universally solvable over $\mathbb{Z}_{2}$. If just $G$ or just $H$ is closed universally solvable, $G \square H$ may or may not be closed universally solvable. For example, $P_{4}$ and $P_{3}$ are closed universally solvable over $\mathbb{Z}_{2}$, and $P_{5}$ and $C_{3}$ are not closed universally solvable over $\mathbb{Z}_{2}$. While $P_{4} \square P_{5}$ is closed universally solvable over $\mathbb{Z}_{2}, P_{3} \square C_{3}$ is not closed universally solvable over $\mathbb{Z}_{2}$. Finally, if neither $G$ nor $H$ is closed universally solvable, $G \square H$ may or may not be closed universally solvable. For example, $P_{5}, P_{8}, C_{3}$, and $W_{4}$, the wheel graph of order 4 , are not closed universally solvable over $\mathbb{Z}_{2}$. While $W_{4} \square P_{8}$ is closed universally solvable over $\mathbb{Z}_{2}, P_{5} \square C_{3}$ is not closed universally solvable over $\mathbb{Z}_{2}$.

Turning to open neighborhood switching, if $G$ and $H$ are both open universally solvable, $G \square H$ may or may not be open universally solvable. For example, the graphs $P_{4}, P_{6}, K_{4}$, and $P_{8}$ are open universally solvable over $\mathbb{Z}_{2}$. While $P_{4} \square P_{6}$ is open universally solvable over $\mathbb{Z}_{2}, K_{4} \square P_{8}$ is not open universally solvable over $\mathbb{Z}_{2}$. If just $G$ or just $H$ is open universally solvable, $G \square H$ may or may not be open universally solvable. For example, $P_{4}$ is open universally solvable over $\mathbb{Z}_{2}$, and $C_{4}$ and $C_{5}$ are not open universally solvable over $\mathbb{Z}_{2}$. While $P_{4} \square C_{4}$ is open universally solvable over $\mathbb{Z}_{2}, P_{4} \square C_{5}$ is not open universally solvable over $\mathbb{Z}_{2}$. Finally, if neither $G$ nor $H$ is open universally solvable over $\mathbb{Z}_{m}$, where $m$ is a power of a prime, then $G \square H$ is not open universally solvable over $\mathbb{Z}_{m}$.

As observed in [9], $\operatorname{det}[A(G \square H)]=\operatorname{det}\left[p_{-B}(C)\right]$, where $B$ is the adjacency matrix of $G, C$ is the adjacency matrix of $H$, and $p_{-B}(x)$ is the characteristic polynomial of $-B$.

THEOREM 5.1. Let $G$ and $H$ be finite, simple graphs, with $|G|=m,|H|=p$, and let $n=m p$. Let $B=A(G)$ and $C=A(H)$. If $G$ and $H$ are not open universally solvable over $\mathbb{Z}_{m}$, where $m$ is a power of a prime, then $G \square H$ is not open universally solvable over $\mathbb{Z}_{m}$.

Proof. Suppose $G$ and $H$ are not open universally solvable over $\mathbb{Z}_{m}$, where $m$ is a power of a prime. Then, $\operatorname{det}(B)=d_{1}$ and $\operatorname{det}(C)=d_{2}$, where $d_{1}$ and $d_{2}$ are nonunits of $\mathbb{Z}_{m}$. Moreover, $p_{-B}(0)=\operatorname{det}\left(0 \cdot I_{m}-(-B)\right)=$ $\operatorname{det}(B)=d_{1}$. Therefore, $p_{-B}(x)=q(x)+d_{1}$ for some $q(x) \in \mathbb{Z}_{m}[x]$ which has a factor of $x$. As every nonunit in a unique factorization ring is nilpotent [3], $d_{1}$ is nilpotent, so there exists a positive integer $j$ such that $d_{1}^{j}=0$. Then, $\left(p_{-B}(x)\right)^{j}=\left(q(x)+d_{1}\right)^{j}=s(x)$ for some $s(x) \in \mathbb{Z}_{m}[x]$ which has a factor of $x$. Then, $s(x)=x \cdot t(x)$ for some $t(x) \in \mathbb{Z}_{m}[x]$. Hence, $\operatorname{det}\left[\left(p_{-B}(C)\right)^{j}\right]=\operatorname{det}[C \cdot t(C)]$. Therefore, $\left[\operatorname{det}\left(p_{-B}(C)\right)\right]^{j}=\operatorname{det}(C) \cdot \operatorname{det}(t(C))=d_{2} \cdot \operatorname{det}(t(C))$. Since $d_{2}$ is a nonunit, and therefore nilpotent, there 
exists a positive integer $k$ such that $d_{2}^{k}=0$. So, $\left[\operatorname{det}\left(p_{-B}(C)\right)\right]^{j k}=d_{2}^{k} \cdot \operatorname{det}(t(C))^{k}=0$, meaning that $\operatorname{det}\left[\left(p_{-B}(C)\right)\right]$ is nilpotent. By Observation $4.5, \operatorname{det}\left[\left(p_{-B}(C)\right)\right]$ is a nonunit of $\mathbb{Z}_{m}$. Thus, $G \square H$ is not open universally solvable over $\mathbb{Z}_{m}$.

Theorem 5.1 does not hold for rings of integers modulo $k$ which are not unique factorization rings, i.e. when $k$ is not a power of a prime. For example, $C_{5}$ and $K_{10}$ are not open universally solvable over $\mathbb{Z}_{6}$, but $C_{5} \square K_{10}$ is open universally solvable over $\mathbb{Z}_{6}$.

Acknowledgments. We wish to thank the College of Saint Benedict \& Saint John's University for providing financial support for this project through a Summer Collaborative Research Grant. We would also like to thank Thomas Sibley for providing a suggestion which helped to broaden the scope of the paper. Finally, we would like to thank the anonymous referee for a careful review of the paper.

\section{REFERENCES}

[1] M. Billis. Unique factorization in the integers modulo m. Am. Math. Monthly, 75:527, 1968.

[2] W.C. Brown. Matrices Over Commutative Rings. Marcel Dekker, New York, 1993.

[3] S. Galovich. Unique factorization rings with zero divisors. Math. Mag., 51(5):276-283, 1978.

[4] A. Giffen and D.B. Parker. On generalizing the 'lights out' game and a generalization of parity domination. Ars Comb., 111:273-288, 2013.

[5] J. Goldwasser and W. Klostermeyer. Odd and even dominating sets with open neighborhoods. Ars Comb., 83:229-247, 2007.

[6] J. Goldwasser, W. Klostermeyer, and G. Trapp. Characterizing switch-setting problems. Linear Multilinear Algebra, 43:121-135, 1997.

[7] R. Hammack, W. Imrich, and S. Klavžar Handbook of Product Graphs. CRC Press, Boca Raton, 2011.

[8] L. Keough and D.B. Parker. An extremal problem for the neighborhood lights out game. Under review.

[9] T. Peters, J. Goldwasser, and M. Young. LIGHTS OUT! on Cartesian products. Electron. J. Linear Algebra, 32:464-474, 2017.

[10] H. Sayama. Estimation of Laplacian spectra of direct and strong product graphs. Disc. Appl. Math., 205:160-170, 2016.

[11] J.R. Silvester. Determinants of block matrices. Math. Gazette, 84(501):460-467, 2000. 